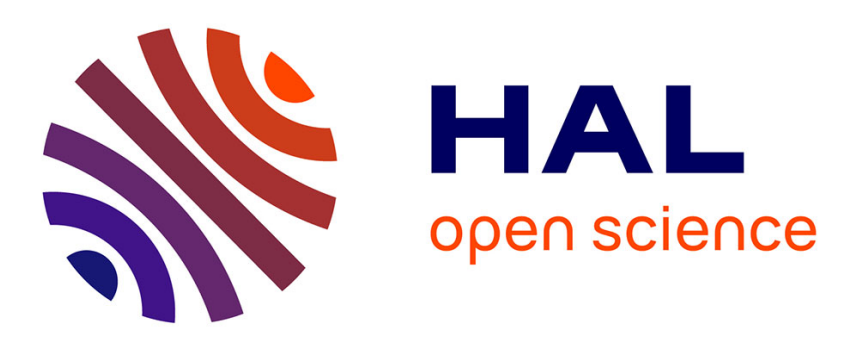

\title{
Nature cristallo-physico-chimique de l'interface entre un semiconducteur et son oxyde propre. II. - Une conception générale de l'interface semiconducteur-oxyde propre \\ O.V. Romanov
}

\section{To cite this version:}

O.V. Romanov. Nature cristallo-physico-chimique de l'interface entre un semiconducteur et son oxyde propre. II. - Une conception générale de l'interface semiconducteur-oxyde propre. Revue de Physique Appliquée, 1984, 19 (5), pp.389-394. 10.1051/rphysap:01984001905038900 . jpa-00245207

\section{HAL Id: jpa-00245207 https://hal.science/jpa-00245207}

Submitted on 1 Jan 1984

HAL is a multi-disciplinary open access archive for the deposit and dissemination of scientific research documents, whether they are published or not. The documents may come from teaching and research institutions in France or abroad, or from public or private research centers.
L'archive ouverte pluridisciplinaire HAL, est destinée au dépôt et à la diffusion de documents scientifiques de niveau recherche, publiés ou non, émanant des établissements d'enseignement et de recherche français ou étrangers, des laboratoires publics ou privés. 


\title{
Nature cristallo-physico-chimique de l'interface entre un semiconducteur et son oxyde propre.
}

\section{II. - Une conception générale de l'interface semiconducteur-oxyde propre ${ }^{*}$ )}

\author{
O. V. Romanov
}

Laboratoire d'Electronique des Corps Solides, Institut de Physique, Université d'Etat de Leningrad, 199 164, Leningrad, URSS

(Reçu le 8 juin 1983, révisé le 21 octobre, accepté le 27 octobre 1983)

\begin{abstract}
Résumé. - On présente une conception générale et originale de la nature cristallo-physico-chimique de l'interface oxyde propre-semiconducteur (OPS) sur la base d'études expérimentales des systèmes OPS formés par les semiconducteurs des groupes $\mathrm{A}^{4}(\mathrm{Si}, \mathrm{Ge})$ et $\mathrm{A}^{3} \mathrm{~B}^{5}(\mathrm{InP}, \mathrm{InAs}, \mathrm{InSb}, \mathrm{GaN}, \mathrm{GaP}, \mathrm{GaAs}, \mathrm{GaSb})$. L'interprétation des résultats expérimentaux est basée sur des considérations de correspondance quasi bidimensionnelle entre le semiconducteur et son oxyde amorphe propre, liés par liaisons chimiques régulières, à travers une couche intermédiaire très mince ( 1 à 3 couches monomoléculaires pour $\mathrm{SiO}_{2}-\mathrm{Si}$ ). La prise en considération d'un paramètre de coïncidence $\left(R=\overline{N_{\mathrm{sc}}^{\mathrm{s}}} / \overline{N_{\mathrm{ox}}^{\mathrm{s}}}\right)$ permet de tirer des conclusions importantes pour tous les semiconducteurs consi-
\end{abstract} dérés :

1) Les oxydes propres forment des couches protectrices sur la surface des semiconducteurs;

2) Il existe toujours des atomes de semiconducteur et d'oxygène non liés (ou liés partiellement) dans la couche intermédiaire, qui peuvent former des centres chargés " génétiques " (intrinsèques) à l'interface OPS

3) Il existe toujours des liaisons chimiques covalentes rompues et non saturées recouvertes par l'oxyde propre à l'interface OPS, qui peuvent former des états rapides d'interface, dont les paramètres $\left(E_{\mathrm{ss}}, N_{\mathrm{ss}}, A_{\mathrm{p}}, A_{\mathrm{n}}\right)$ sont déterminés soit par l'interaction coulombienne avec les centres chargés de la couche intermédiaire (partie continue du spectre $N_{\mathrm{ss}}(E)$ ), soit par la liaison chimique avec des impuretés de nature "biographique» (par exemple des métaux $\mathrm{Cu}, \mathrm{Ag}, \mathrm{Au}, \mathrm{Rh}, \mathrm{Ru}, \mathrm{Pt}, \mathrm{Fe}, \mathrm{Co}, \mathrm{Ni}$ etc.) qui représentent la partie discrète du spectre $N_{\mathrm{ss}}(E)$.

L'interprétation générale de la nature de l'interface OPS prend en considération simultanément trois aspects principaux de l'interaction des deux substances : les aspects cristallographique, chimique et physique.

\begin{abstract}
The results of experiments carried out in the electrolyte-own oxide-semiconductor systems, with semiconductors selected from groups $\mathrm{A}^{4}\left(\mathrm{Si}, \mathrm{Ge}\right.$, with surfaces (100) and (111)), and $\mathrm{A}^{3} \mathrm{~B}^{5}(\mathrm{InP}, \operatorname{InAs}, \operatorname{InSb} ; \mathrm{GaN}$, $\mathrm{GaP}, \mathrm{GaAs}, \mathrm{GaSb}$ with surfaces (111)- $\mathrm{A}^{3}$ and (111)- $\left.\mathrm{B}^{5}\right)$ in different aqueous electrolytes have made it possible to establish the cristallo-physico-chemical nature and the main rules governing the formation of a double electrical layer, and also to determine other electrophysical properties of the interface between a semiconductor and its own oxide irrespective of the method used to form it. The results obtained have been interpreted supposing a quasi-two-dimensional structural and chemical correspondence between the contact-monolayers of the semiconductor matrix and the growing insulator, in the case of an ordering interaction (caused by the chemical binding forces). The first approach of a general theory of the interface own oxide-semiconductor takes into account the three important aspects of this interface : structural, physical and chemical aspects, the details of which are here presented.
\end{abstract}

\section{Introduction.}

Les résultats des expériences systématiques effectuées sur des semiconducteurs monocristallins bien choisis $\left(\mathrm{A}^{4}: \mathrm{Si}, \mathrm{Ge}\right.$; et $\mathrm{A}^{3} \mathrm{~B}^{5}:$ InP, InAs, InSb, GaN, GaP,

$\left(^{*}\right)$ Cet article a été rédigé alors que l'auteur était en visite au laboratoire de Physique des Composants à Semiconducteurs (ERA CNRS No 659) de l'ENSERG, Institut National Polytechnique de Grenoble.
GaAs, GaSb) [1] nous permettent de formuler les règles générales de formation d'une double couche électrique à l'ïnterface oxyde propre-semiconducteur (OPS) lors de l'étape initiale de croissance de l'oxyde propre $\left(d_{\text {ox }} \leqslant 50 \AA\right)$. Ces résultats constituent nos bases d'élaboration d'une conception générale approchée de l'interface OPS. Ces règles sont les suivantes :

1) En l'absence d'impuretés la charge électrique localisée dans l'oxyde propre a une nature "géné- 
tique », qui est parfaitement déterminée par le processus d'oxydation lui-même;

2) Cette charge électrique est localisée généralement dans la partie (étroite) la plus proche de l'oxyde propre $\left(\Delta \simeq 7\right.$ à $16 \AA$ pour $\mathrm{Si}^{-S_{i O}}$ et $\Delta \leqslant 50 \AA$ pour les autres semiconducteurs étudiés) de l'interface OPS :

3) Dans les mêmes conditions la valeur algébrique de cette charge dépend de la nature chimique du semiconducteur et change régulièrement selon la classification des semiconducteurs des groupes $\mathrm{A}^{4}$ et $\mathrm{A}^{3} \mathrm{~B}^{5}$

4) Toujours dans les mêmes conditions, pour un même semiconducteur, la valeur algébrique de cette charge dépend clairement de l'orientation cristallographique de la surface étudiée;

5) L'introduction d'impuretés lors du processus de formation de l'interface OPS joue un rôle important pour la double couche électrique et les paramètres électrophysiques de cette interface.

De notre point de vue, ces règles générales de formation de la double couche électrique à l'interface OPS révèlent trois aspects importants de sa nature : cristallographique, physique et chimique. Dans cette deuxième partie nous essayerons de réunir ces trois aspects dans une conception générale approchée de l'interface OPS.

\section{Nature de la couche intermédiaire et de la double couche électrique à l'interface OPS.}

La notion de couche intermédiaire à l'interface OPS joue un rôle important dans la compréhension de l'interface OPS $[2,3]$. Jusqu'à présent sa nature et les règles de sa formation restaient insuffisamment comprises [2 à 5].

La nature génétique et la localisation d'une partie importante de la charge électrique de l'oxyde propre au voisinage de l'interface OPS nous permettent de supposer l'existence de centres génétiques (intrinsèques) d'ionisation ou de capture électronique dans une couche assez mince qui sépare le réseau cristallin du semiconducteur et la couche amorphe d'oxyde propre. La nature de cette couche intermédiaire et de ses centres électriques est liée étroitement au mécanisme de croissance de l'oxyde propre sur la surface des semiconducteurs. Selon les résultats de divers auteurs [6 à 9], ce mécanisme se ramène au transport des composés oxydants à travers l'oxyde propre vers l'interface OPS ou à la diffusion de particules neutres (oxydation thermique), ou au déplacement d'ions par le champ électrique (oxydation anodique). Dans tous les cas pendant la croissance d'oxyde propre nous observons la transformation successive de couches monoatomiques du réseau cristallin semiconducteur en des couches monomoléculaires d'oxyde propre, dont la constitution chimique est déterminée par les propriétés chimiques des composants de cette réaction.
Selon les principes donnés par G. A. Samorjai [10] la formation des premières couches atomo-moléculaires sur les surfaces de corps monocristallins par adsorption chimique est toujours soumise à des règles, qui expriment l'action régulière du réseau sur la couche monoatomique (ou mono-moléculaire) de la substance adsorbée : la règle de compacité maximale, la règle de conservation de symétrie de rotation et la règle d'identité des vecteurs des cellules élémentaires. Dans le cas d'oxydation de la couche superficielle monoatomique d'un semiconducteur monocristallin, qui est lié par des liaisons covalentes précisément orientées dans l'espace avec la couche suivante, le phénomène se réduit à la formation d'une substance chimique nouvelle et différente du réseau du point de vue cristallographique, chimique et physique. C'est un oxyde propre amorphe qui a une structure cristallographique désordonnée, basée sur la cellule élémentaire tétrahédrique $\mathrm{SiO}_{4}[2$ à $4,7,11]$, mais qui en même temps, reste lié par des liaisons déjà ionocovalentes avec le réseau du semiconducteur. Il s'en suit que ces liaisons de la frontière OPS doivent posséder une régularité cristallographique due à la couche superficielle du réseau cristallin du semiconducteur. Cela nous permet de constater la régularité structurelle de la couche intermédiaire du système OPS. L'épaisseur de cette couche est déterminée par la courte portée d'une liaison chimique et ne comprend que 1 à 3 couches monomoléculaires de l'oxyde propre. Autrement dit, lors du processus d'oxydation d'un semiconducteur, nous avons renouvellement permanent d'une couche intermédiaire assez mince et régulière qui sépare le réseau monocristallin du semiconducteur et l'oxyde propre amorphe. La régularité de cette couche intermédiaire eșt déterminée précisément d'une part par l'orientation cristallographique de la surface du semiconducteur et d'autre part, par les dimensions de la cellule élémentaire de l'oxyde propre amorphe. En plus, il est important de souligner que la couche intermédiaire du système OPS représente la zone où s'effectue la réaction chimique de transformation du semiconducteur en oxyde propre quel que soit le mécanisme de transport des ingrédients de cette réaction à travers l'oxyde. Cela veut dire qu'on aura une concentration élevée de différents défauts intrinsèques (ou génétiques) dans cette région.

En première approximation on peut évaluer quantitativement la correspondance cristallo-chimique à travers la couche intermédiaire des réseaux cristallins des divers semiconducteurs avec leurs oxydes propres amorphes $[12,13]$. Compte tenu de la nature cristallophysico-chimique de la transformation de la couche superficielle monoatomique du semiconducteur en une couche monomoléculaire et régulière d'oxyde propre dans le champ de forces chimiques de courte portée, il faut comparer les valeurs moyennes des densités atomiques superficielles du réseau semiconducteur $\left(\overline{N_{\mathrm{sc}}^{\mathrm{s}}}\right)$ et de l'oxyde propre $\left(\overline{N_{\mathrm{ox}}^{\mathrm{s}}}\right)$. On peut 
Tableau I. - Comparaison des paramètres "électroniques " (affinité $\chi$; électronégativité $K$; potentiel d'ionisation $V_{\mathrm{i}}$ ) et "géométriques" (rayon covalent $r_{\mathrm{K}} ;$ constante du réseau a ; paramètre $R=\overline{N_{\mathrm{sc}}^{\mathrm{s}}} \overline{N_{\mathrm{ux}}^{\mathrm{s}}} d e \mathrm{Si}$ et de $\mathrm{Ge}$ avec la charge de la double couche électrique $Q_{v x}^{0}$ à l'interface OPS.

[Comparison of some " electronic" (affinity $\chi$; electronegativity $K$; ionisation potential $V_{\mathrm{i}}$ ) and " geometrical " (covalent radius $r_{K}$; lattice constant $\mathrm{a}$; parameter $\left.R=\overline{N_{\mathrm{sc}}^{\mathrm{s}}} / \overline{N_{\mathrm{vx}}^{\mathrm{s}}}\right)$ parameters of $\mathrm{Si}$ and $\mathrm{Ge}$, with the electric charge $Q_{\mathrm{vx}}^{0}$ of the double layer at the interface.]

\begin{tabular}{|c|c|c|c|c|c|c|c|}
\hline \multirow[b]{2}{*}{ Surface } & \multicolumn{3}{|c|}{$\begin{array}{l}\text { Paramètres } \\
\text { "géométriques" }\end{array}$} & \multicolumn{3}{|c|}{$\begin{array}{l}\text { Paramètres } \\
\text { "électroniques" }\end{array}$} & \multirow{2}{*}{$\begin{array}{l}a_{0 x}^{0} \cdot 10^{-11} \\
\text { el.cm-2 }\end{array}$} \\
\hline & $r_{k}(\dot{A})$ & I $(\dot{A})$ & $\bar{N}_{s i}^{3} / \bar{N}_{o x}^{3}$ & $x(e v)$ & $k$ & $V_{i}^{*}(e V)$ & \\
\hline $\begin{array}{l}\text { Si (1111) } \\
\text { Si (100) }\end{array}$ & \} .17 & 5.4304 & 1,69 & 4,05 & 1,8 & & $\begin{array}{l}+14 \text { ذ } 14) \\
+(1 \text { ذ } 5)\end{array}$ \\
\hline $\begin{array}{l}\text { Ge (1111) } \\
\text { Ge (100) }\end{array}$ & \}, 22 & 5,6575 & 1,49 & 4.1 & 1,7 & 7,88 & $\begin{array}{l}+10,5 \text { ذ } 0,71 \\
+(0,3 \text { ذ } 0,4)\end{array}$ \\
\hline
\end{tabular}

les calculer selon les formules suivantes :

$$
\begin{aligned}
& \overline{N_{\mathrm{sc}}^{\mathrm{s}}}=\left(\frac{N_{\mathrm{a}} K \rho_{\mathrm{sc}}}{M_{\mathrm{sc}}}\right)^{2 / 3}, \text { pour } \mathrm{A}^{4} \text { et } \mathrm{A}^{3} \mathrm{~B}^{5} \\
& \overline{N_{\mathrm{ox}}^{\mathrm{s}}}=\left(\frac{N_{\mathrm{a}} n_{\mathrm{ox}} \rho_{\mathrm{ox}}}{M_{\mathrm{ox}}}\right)^{2 / 3}, \text { pour } \mathrm{A}^{4} \\
& \overline{N_{\mathrm{ox}}^{\mathrm{s}}}=\left(\frac{M_{\mathrm{ox}_{1}}}{2 N_{\mathrm{a}} \rho_{\mathrm{ox}_{1}} n_{\mathrm{ox}_{1}}}+\frac{M_{\mathrm{ox}_{2}}}{2 N_{\mathrm{a}} \rho_{\mathrm{ox}_{2}} n_{\mathrm{ox}_{2}}}\right)^{-2 / 3}, \\
& \text { pour } \mathrm{A}^{3} \mathrm{~B}^{5}
\end{aligned}
$$

où $N_{\mathrm{a}}$ est le nombre d'Avogadro, $K=1$ pour $\mathrm{A}^{4}$ et $K=2$ pour $\mathrm{A}^{3} \mathrm{~B}^{5} ; \rho_{\mathrm{sc}}$ et $M_{\mathrm{sc}}$ : densité et masse moléculaire du semiconducteur; $n_{\mathrm{ox}}, n_{\mathrm{ox}}, n_{\mathrm{ox} 2}:$ nombre d'atomes du réseau dans la molécule d'oxyde propre; $M_{\mathrm{ox}}, M_{\mathrm{ox}_{1}}, M_{\mathrm{ox}_{2}}, \rho_{\mathrm{ox}}, \rho_{\mathrm{ox}_{1}}, \rho_{\mathrm{ox}_{2}}$, paramètres correspondant aux oxydes propres les plus probables.

Introduisons un paramètre de correspondance cristallo-physico-chimique du réseau cristallin du semiconducteur à son oxyde propre amorphe :

$$
R=\frac{\overline{N_{\mathrm{sc}}^{\mathrm{s}}}}{\overline{N_{\mathrm{ox}}^{\mathrm{s}}}} ;
$$

Les calculs de ce paramètre $R$ peuvent être effectués en utilisant des résultats d'études de la nature chimique des oxydes minces propres anodiques de différents semiconducteurs, obtenus par spectroscopie Auger, SIMS, ESCA, etc. $[14,15]$; ces résultats montrent, que les oxydes de nos matériaux comprennent $\mathrm{SiO}_{2}, \mathrm{GeO}_{2}, \mathrm{In}_{2} \mathrm{O}_{3}, \mathrm{As}_{2} \mathrm{O}_{3}, \mathrm{Sb}_{2} \mathrm{O}_{3}, \mathrm{Ga}_{2} \mathrm{O}_{3}$ et $\mathrm{P}_{2} \mathrm{O}_{5}$. Nos calculs sont présentés dans les tableaux 1 , 2,3 . Il s'ensuit que dans tous les cas nous avons l'inégalité :

$$
R>1
$$

qui conduit aux conclusions importantes suivantes pour une conception générale et un modèle cristallophysico-chimique de l'interface OPS (Fig. 1) :

1) Au cours de la formation de la monocouche d'oxyde propre, quand la couche monoatomique du semiconducteur se transforme en monocouche d'oxyde propre, certains atomes supplémentaires suprastoechiométriques du semiconducteur ne sont pas liés (ou sont liés partiellement) au réseau cristallin assez régulier dans la couche intermédiaire d'oxyde propre. Notons que ce résultat a été observé pour l'oxyde thermique du silicium $[3,4,16,17]$.

2) Le paramètre $R$ caractérise la différence de stoechiométrie entre la couche intermédiaire et l'oxyde volumique pour chaque semiconducteur (voir Tableaux I à III). Il varie régulièrement pour une même colonne de composés :

$$
\begin{aligned}
R(\mathrm{Si}) & >R(\mathrm{Ge})>1 \\
R(\mathrm{InP}) & >R(\mathrm{InAs})>R(\mathrm{InSb})>1 \\
R(\mathrm{GaN}) & >R(\mathrm{GaP})>R(\mathrm{GaAs})>R(\mathrm{GaSb})>1 .
\end{aligned}
$$

3) La faible épaisseur et la régularité de la couche intermédiaire étant déterminées par le réseau cristallin du semiconducteur, ceci explique la dépendance du paramètre $R$ avec les diverses orientations cristallographiques des surfaces d'un même semiconducteur. Pour les surfaces $(100)$ et (111) cette dépendance a une nature purement cristallographique, liée à la densité superficielle des atomes du réseau cristallin du semiconducteur. Cela veut dire que la densité d'atomes suprastoechiométriques dans la couche intermédiaire sera plus grande pour la surface (111), que pour la surface (100). Le même résultat a été trouvé expérimentalement pour Si [17].
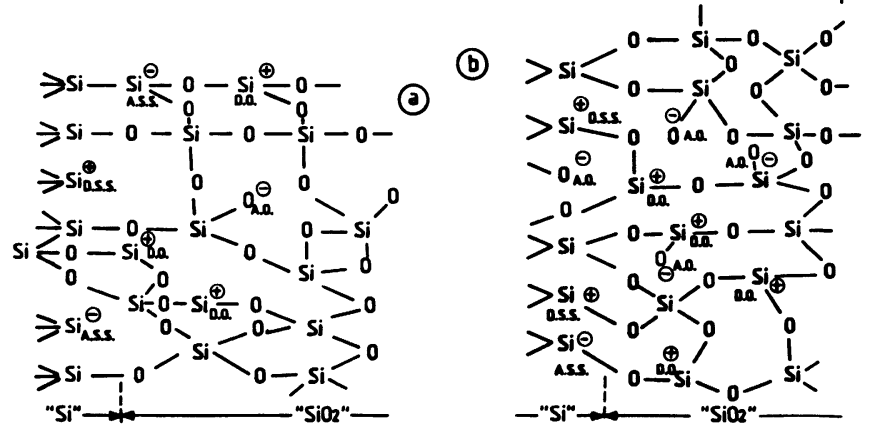

Fig. 1. - Modèle cristallo-physico-chimique approché de l'interface OPS pour deux orientations de la surface de $\mathrm{Si}$ : (a) = (111); (b) = (100). ASS = états de surface de type accepteur; DSS = états de surface de type donneur; AO $=$ accepteur en oxyde $; D O=$ donneur en oxyde; $\mathrm{AO}$ et DO-centres chargés génétiques.

[Approximate scheme of the semiconductor ( $\mathrm{Si}$ )-oxide interface for two different surface orientation of Si. (a) $=(111)$ : (b) $=(100)$; ASS $=$ acceptor-like surface states; DSS = donor-like surface states; $\mathrm{AO}=$ oxide acceptor $; \mathrm{DO}=$ oxide donor.] 
4) Dans le cas des surfaces (111)- $\mathrm{A}^{3}$ et (111)- $\mathrm{B}^{5}$ pour lesquelles $R(111)=R(\overline{111})$ la distribution des atomes suprastoechiométriques dans la couche intermédiaire peut être différente (Fig. 2), de même que la polarité des liaisons chimiques entre le semiconducteur et la couche intermédiaire.

5) Par suite de la non-correspondance cristallochimique (entre le semiconducteur et l'oxyde propre) traduite quantitativement par l'inégalité (5), il existe des liaisons covalentes rompues et non saturées des atomes du réseau à l'interface ("dangling bonds") couvertes par l'oxyde propre.

6) La conservation de la stoechiométrie exacte de l'oxyde propre hors de la couche intermédiaire. révélée par plusieurs résultats expérimentaux $[4,5$, $18,19]$, peut être interprétée en tenant compte de la présence permanente d'atomes d'oxygène non liés (ou liés partiellement) dans la couche intermédiaire.

7) Il est évident que des impuretés extrinsèques peuvent être présentes dans la couche intermédiaire, de même que sur la frontière de cette couche avec le réseau cristallin du semiconducteur [20 à 23].

Ainsi les particularités principales de formation de l'oxyde propre dans le processus d'oxydation d'un semiconducteur déterminent l'existence de la couche intermédiaire à l'interface OPS qui est le siège de divers défauts intrinsèques, dont les principaux sont les excès suprastoechiométriques d'atomes du réseau cristallin du semiconducteur et d'atomes d'oxygène (ou autre oxydant) non liés ou liés partiellement au réseau quasi régulier de la couche intermédiaire très mince ( 7 à $16 \AA$ pour $\left.\mathrm{Si}-\mathrm{SiO}_{2}\right)$. En particulier, en l'absence d'impuretés, ces atomes jouent le rôle d'accepteurs et de donneurs, et donnent à la couche intermédiaire les propriétés d'un isolant autocompensé d'à peu près deux dimensions. En présence d'impuretés on aura pénétration dans cette couche des impuretés extrinsèques dont les dimensions atomiques sont comparables aux paramètres cristallo-

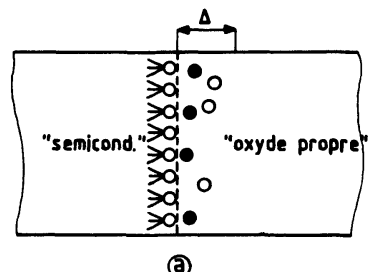

(2)

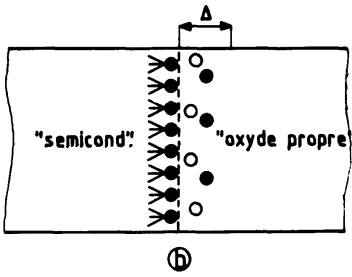

Fig. 2. - Influence de l'axe polaire [111] dans les semiconducteurs $A^{3} B^{5}$ sur le modèle de l'interface OPS : $\mathrm{a}$ - surface (111)- $\mathrm{A}^{3} ; \mathrm{b}$ - surface (111)- $\mathrm{B}^{5} ; \Delta=$ couche intermédiaire; $O$ et $\bullet$ atomes $A^{3}$ et $B^{5}$ suprastoechiométriques de la matrice dans la couche intermédiaire.

[Influence of the polar axis [111] direction in $\mathrm{A}^{3} \mathrm{~B}^{5}$ semiconductors on the interface model; $a: A^{3}(111)$ surface; $\mathrm{b}: \mathrm{B}^{5}(\overline{1} \overline{1})$ surface. $\Delta=$ intermediate layer; $O$ and $\bullet$ : $A^{3}$ and $B^{5}$ suprastoechiometric atoms of the matrix in the intermediate layer.] graphiques de la couche intermédiaire quasi régulière. Par exemple, pour le système $\mathrm{Si}_{-} \mathrm{SiO}_{2}$, ce peut être $\mathrm{Cl}, \mathrm{F}, \mathrm{Na}, \mathrm{K}, \mathrm{H}$, etc. Par suite de l'ionisation des défauts intrinsèques et extrinsèques de la couche intermédiaire par échange électronique tunnel avec les bandes de conduction et de valence du semiconducteur, on a formation de la charge $Q_{\mathrm{ox}}$ (Fig. 3), dont la grandeur algébrique est donnée par :

$$
Q_{\mathrm{ox}}=q \int_{0}^{\infty}\left(\sum_{i} N_{\mathrm{D} i}(E) f_{\mathrm{D} i}(E)-\sum_{k} N_{\mathrm{A} k} \cdot f_{\mathrm{A} k}(E)\right) \mathrm{d} E
$$

où $N_{\mathrm{D} i}$ et $N_{\mathrm{A} k}$ sont les densités superficielles et énergétiques de donneurs et d'accepteurs de la couche intermédiaire; $f_{\mathrm{Di}}$ et $f_{\mathbf{A} k}$, les fonctions de Fermi-Dirac correspondantes, dont l'expression fait intervenir les niveaux d'énergies $E_{\mathrm{D} i}$ et $E_{\mathrm{A} k}$. A leur tour les niveaux d'énergie $E_{\mathrm{D} i}$ et $E_{\mathrm{A} k}$ et la charge électrique des niveaux accepteurs et donneurs correspondants seront déterminés par les paramètres physico-chimiques du système OPS : les largeurs des bandes interdites du semiconducteur $\left(E_{\mathrm{g}}^{\mathrm{sc}}\right)$ et de l'oxyde propre $\left(E_{\mathrm{g}}^{\mathrm{ox}}\right)$, leurs permittivités électriques $\left(\varepsilon_{\mathrm{sc}}, \varepsilon_{\mathrm{ox}}\right)$, les potentiels d'ionisation $\left(V_{\mathrm{i}}^{+}\right)$et d'affinité $\left(V_{\mathrm{i}}^{-}\right)$des atomes (des défauts) correspondants. D'une manière générale, on sait que les éléments, dont le potentiel d'ionisation est faible ( $\mathrm{Si}, \mathrm{Ge}, \mathrm{In}, \mathrm{Ga}, \mathrm{Al}, \mathrm{B}, \mathrm{N}, \mathrm{K}$, etc.) jouent le rôle de donneur dans la couche intermédiaire, et que

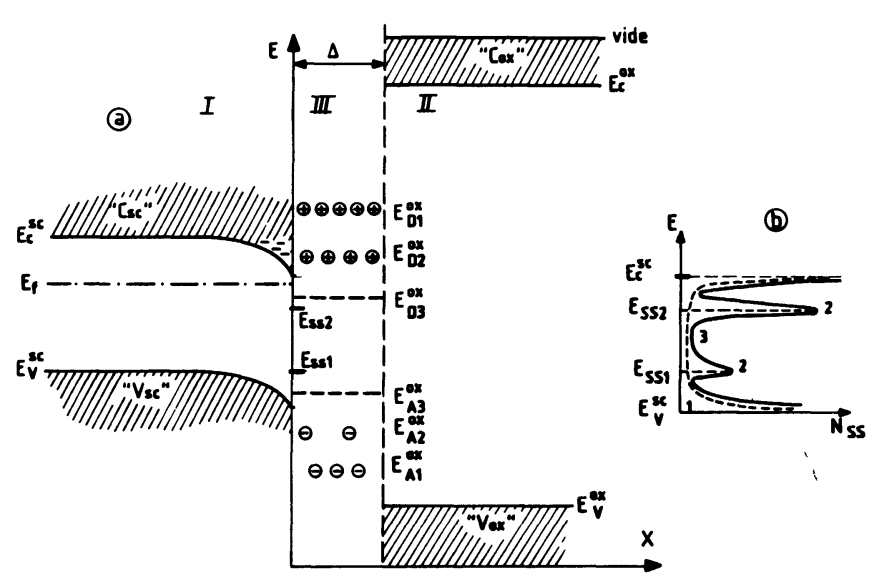

Fig. 3. - Schéma énergétique de l'interface OPS (a) et distribution énergétique des états d'interface dans la bande interdite du semiconducteur (b). I : semiconducteur; II : oxyde amorphe propre; III : couche intermédiaire de largeur $\triangle$; Parties continues (1) et discrètes (2) du spectre total (3 : trait plein) des états d'interface. $E_{\mathrm{D} i}^{\mathrm{ox}}$ et $E_{\mathrm{A} k}^{\mathrm{ox}}$ : niveaux donneurs et accepteurs dans la couche intermédiaire.

[Interface energetic diagram (a) and (b) interface state energy distribution inside the semiconductor bandgap. (I) : semiconductor; (II) own amorphous oxide; (III) : intermediate layer. Continuous (1) or discret (2) parts of the whole distribution $(3:$ full line) of the interface states. $E_{\mathrm{D} i}$ and $E_{\mathrm{A} k}$ : donor and acceptor levels in the intermediate layer.] 
Tableau II. - Paramètres de l'interface OPS sur la surface (111) $\mathrm{B}^{5}$ du sous-groupe $\operatorname{In} \mathrm{B}^{5}$ de $\mathrm{A}^{3} \mathrm{~B}^{5}$ (solution $\mathrm{NaOH})$.

[Interface parameters for a $\mathrm{B}^{5}(111)$ surface of the InB $^{5}$ group $(\mathrm{NaOH}$ solution).]

\begin{tabular}{|c|c|c|c|}
\hline Paramètres & $n-\ln P$ & $n-\ln A s$ & $p-\ln S b$ \\
\hline$\overline{N_{s c}^{s}} / \overline{N_{o x}^{s}}$ & 1,29 & 1.20 & 1,08 \\
\hline$V_{s}^{0}(V)$ & $+(0,09$ ذ̀ 0,11$)$ & $+10,03$ à 0,06 & $-(0,09$ à 0,11$)$ \\
\hline$a_{0 x}^{0}($ el.cm-2) & + (7 à 12). $10^{11}$ & $+(1,5$ à 3$) \cdot 10^{11}$ & $-(8$ à 10$) .10^{11}$ \\
\hline $\begin{array}{l}\text { Potentiels } \\
\text { d'ionisation } V_{i}^{+}(V)\end{array}$ & $\begin{array}{l}V_{i}^{*}(l n)=-5,78 \\
V_{i}^{*}(P)=-10,55\end{array}$ & $V_{i}^{*}(A s)=-9,81$ & $V_{i}^{*}(S b)=-8,64$ \\
\hline Comparaison de $a_{0 x}^{\circ}$ & \multicolumn{3}{|c|}{$a_{o x}^{0}(\ln P)>a_{o x}^{0}(\ln A s)>0>a_{o x}^{0}(\ln S b)$} \\
\hline
\end{tabular}

les éléments qui ont une affinité importante $(\mathrm{O}, \mathrm{Cl}$. $\mathrm{F}, \mathrm{P}, \mathrm{H}$, etc.) jouent le rôle d'accepteur.

Ainsi, en variant les conditions cristallo-physicochimiques de formation de l'interface OPS, on peut faire varier la grandeur algébrique de la charge $Q_{\mathrm{ox}}$. Il s'en suit qu'en l'absence d'impuretés il doit en général exister une corrélation entre les variations de $Q_{\text {ox }}$ et celles du paramètre $R$ de l'interface OPS dans chaque groupe de semiconducteurs. Les résultats des tableaux I à III nous le montrent pour les semiconducteurs des groupes $\mathrm{A}^{4}$ et $\mathrm{A}^{3} \mathrm{~B}^{5}$ dans des conditions identiques.

\section{Nature des états de surface et de leur distribution énergétique.}

La nature cristallo-physico-chimique des états de surface à l'interface OPS est déterminée en même temps par les liaisons covalentes rompues à la frontière du réseau cristallin (conséquence de (5)) et par le réseau quasi bi-dimensionnel $(\Delta \simeq 7$ à $16 \AA)$ des donneurs et des accepteurs chargés, distribués au hasard dans la couche intermédiaire (Figs. 1 à 3). Les états de surface peuvent être localisés sur ces liaisons rompues recouvertes par l'oxyde propre. Mais par suite de l'étroite proximité de ces liaisons non saturées avec l'ensemble des charges (positives $-N_{\mathrm{D} i}$ et négatives $-N_{\mathrm{A} k}$ ) qui forment $Q_{\mathrm{ox}}$, les paramètres principaux des états de surface correspondants seront déterminés par le champ électrique local, créé par les donneurs $\left(N_{\mathrm{D} i}\right)$ et accepteurs $\left(N_{\mathrm{A} k}\right)$ les plus proches dans la couche intermédiaire. $\mathrm{La}$ distribution aléatoire de $N_{\mathrm{D} i}$ et $N_{\mathrm{A} k}$ dans la couche intermédiaire et la variation statistique de la grandeur absolue et du signe du champ électrique local sur les liaisons non saturées, produisent la partie continue de la distribution énergétique des états de surface dans le système OPS, de même que sur la surface "réelle» des semiconducteurs. Les paramètres électrophysiques de ce genre d'états de surface (la densité et la distribution en énergie $N_{\text {ss }}(E)$, leur type, les sections de capture des trous et des électrons, etc.) dépendent de la nature cristallo-physico-chimique et de la structure microscopique de la couche intermédiaire à l'interface OPS (Figs. 1 à 3). Les autres types d'états de surface, à distribution énergétique discrète, proviennent de l'adsorption des microimpuretés de métaux lourds, comme $\mathrm{Cu}, \mathrm{Ag}, \mathrm{Au}, \mathrm{Fe}, \mathrm{Ni}, \mathrm{Co}, \mathrm{Pt}$, $\mathrm{Rh}$, etc. sur la surface des semiconducteurs. Leur nature et leurs paramètres électriques sont déterminés par la liaison chimique saturée entre un atome de semiconducteur à l'interface OPS et un atome de métal. Ceci explique l'augmentation de la densité de ces états de surface avec la quantité de métal ađsorbée [20 à 24], sans variation importante de la position énergétique de leurs niveaux. De plus, leur apparition dépend de la possibilité pour les atomes métalliques de gagner l'interface OPS à travers l'oxyde propre. Cette possibilité dépend elle-même de la solubilité de l'oxyde propre et des dimensions géométriques de l'atome adsorbé (rayons covalent ou ionique) et de la cellule élémentaire de l'oxyde propre. La condition nécessaire et suffisante pour la formation d'états de surface de ce genre est le dégagement d'un atome neutre de métal $\left(\mathrm{M}_{\mathrm{e}}^{+n}+n \mathrm{e}^{-} \rightarrow \mathrm{M}^{0}\right)$ sur les liaisons covalentes non saturées de l'interface OPS [20].

\section{Conclusions.}

En présentant cette approche d'une conception générale de l'interface OPS et son modèle microscopique possible nous voudrions souligner que cette conception est basée sur des considérations assez générales prenant en compte trois aspects principaux d'interaction qui se déroulent au cours de la transformation $\mathrm{du}$ réseau monocristallin du semiconducteur en oxyde propre : les aspects cristallographique, physique et chimique. Pour cette première approche nous avons dû simplifier la situation réelle par suite du manque de résultats systématiques et sûrs relatifs aux différents types d'interaction cristallo-physicochimique sur l'interface OPS, en ne conservant que les idées principales.

Tableau III. - Paramètres de l'interface OPS sur la surface du sous-groupe $\mathrm{GaB}^{5}$ de $\mathrm{A}^{3} \mathrm{~B}^{5}$ (solution $\mathrm{Na}_{2} \mathrm{SO}_{4}$ ).

[Interface parameters of the $\mathrm{GaB}^{5}$ surface $\left(\mathrm{Na}_{2} \mathrm{SO}_{4}\right.$ solution).]

\begin{tabular}{|c|c|c|c|c|}
\hline Paramètres & $n$-GaN & $n$-GaP & n-GaAs & $n-G a S b$ \\
\hline$\overline{N_{s c}^{s}} / \overline{N_{o x}^{s}}$ & 1,71 & 1,37 & 1.27 & 1,12 \\
\hline $\begin{array}{l}\text { Potentiels } \\
\text { d'ionisation } V_{i}^{+}(V)\end{array}$ & $\begin{array}{l}v_{i}^{*}(G)=-6,00 \\
V_{i}^{*}(N)=-14,54\end{array}$ & $V_{i}^{*}(P)=-10,55$ & $V_{i}^{*}(A s)=-9,81$ & $V_{i}^{*}(S b)=-8,64$ \\
\hline $\begin{array}{l}\text { Comparaison } \\
\text { de } a_{\text {ox }}^{0}\end{array}$ & & & $a_{o x}^{0}$ (GaAs) & $a_{o x}^{0}(G a S b)$ \\
\hline
\end{tabular}


L'aspect cristallographique intervient par la correspondance des structures du réseau monocristallin de semiconducteur et de l'oxyde propre amorphe en volume et régulier dans la couche intermédiaire. De plus, il faut prendre en considération la perméabilité géométrique (selon les dimensions de la cellule cristalline) de la couche de l'oxyde propre pour des impuretés de divers rayons covalents (ou ioniques). Le phénomène de polymorphisme des oxydes possibles $\left(\mathrm{GeO}_{2}, \mathrm{SiO}_{2}, \mathrm{In}_{2} \mathrm{O}_{3}, \mathrm{Ga}_{2} \mathrm{O}_{3}, \mathrm{Sb}_{2} \mathrm{O}_{3}\right.$, etc...) complique cet aspect.

L'aspect chimique est déterminé par la nature chimique (polyvalence, amphotéricité, acidité, basicité, stoechiométrie, solubilité pour le cas d'oxydes anodiques ou sublimation pour le cas d'oxydes thermiques, polymorphisme, etc...) des participants à la réaction chimique (ou électrochimique) et des impuretés possibles. Il faut aussi prendre en considération la thermodynamique des réactions chimiques principales et parallèles possibles, ce qui peut être assez important dans le cas des semiconducteurs complexes $\left(\mathrm{A}^{3} \mathrm{~B}^{5}, \mathrm{~A}_{x}^{3} \mathrm{~A}_{1-x}^{\prime 3}, \mathrm{~B}^{5}, \mathrm{~A}^{3} \mathrm{~B}_{y}^{\prime 5} \mathrm{~B}_{1-y}^{5}\right.$, etc...).

L'aspect physique est déterminé par la structure énergétique de l'hétérojonction abrupte oxyde propre - semiconducteur et par les paramètres électrophysiques des états de surface (densité superficielle, distribution en énergie, états de charge. sections de capture des électrons et des trous), ainsi que par les états électroniques dans la couche intermédiaire et dans le volume de l'oxyde propre, constitués par les défauts intrinsèques ou extrinsèques, dont la nature a été prise en considération.

On peut penser que le problème scientifique et technique (microélectronique, corrosion des corps solides, catalyse hétérogène, couches de défense, etc...) de la formation de l'interface oxyde propre - semiconducteur trouvera sa solution au fur et à mesure que l'on approfondira la compréhension de sa nature cristallo-physico-chimique.

\section{Remerciements.}

Je remercie beaucoup M. A. Chovet, enseignant à l'ENSERG (Institut National Polytechnique de Grenoble) pour son aide lors de la rédaction de ce texte. Je remercie d'autre part MM. G. Kamarinos, G. Pananakakis, A. Chovet et S. Cristoloveanu (du laboratoire de Physique des Composants à Semiconducteurs de l'ENSERG) pour les utiles discussions concernant le thème de cette publication.

\section{Bibliographie}

[1] Romanov, O. V., Revue Phys. Appl. (article proposé : Partie I)

[2] Litovchenko, V. G., Sov. Phys. Semicond. 6 (1972) 802-811.

[3] Goetzberger, A., Klausmann, E., Schulz, M. J., CRC Crit. Rev. in Solid State Science 6 (1976) $1-56$.

[4] Pantelides, S. T., The Physics of $\mathrm{SiO}_{2}$ and its interfaces (Pergamon Press, New York) 1978.

[5] Helms, C. R., J. Vac. Sci. Technol. 16 (1979) 608-614.

[6] Ligenza, J. K., Spitzer, W. G., J. Phys. Chem. Sol. 14 (1960) 131.

[7] Deal, B. E., J. Electrochem. Soc. 121 (1974) 198 C$202 \mathrm{C}$.

[8] Pliskin, W. A., Gnall, R. P., J. Electrochem. Soc. 111 (1964) 872-880.

[9] Mackintosh, W. D., Platner, H. P., J. Electrochem. Soc. 127 (1977) 396-402.

[10] SamorjaI, G. A., Principles of surface chemistry (Prentice-Hall, New York) 1972.

[11] Pauling, L., The Nature of the Chemical bond (Cornell Univ. press, New York) 1948.

[12] Romanov, O. V., 4e Colloque sur la physico-chimie des surfaces de semiconducteurs, Acad. Sci. de URSS, Novosibirsk (1981).

[13] Romanov, O. V., Sov. Phys. Semicond. 16 (1982) 266-270.
[14] Belyi, V. T., Smirnova, T. P., Golubenko, A. N. 4e Colloque sur la physico-chimie des surfaces de semiconducteurs, Acad. Sci. d'URSS, Novosibirsk (1981).

[15] Wilmsen, C. W., J. Vac. Sci. Technol. 19 (1981) 279. 281.

[16] Raider, S. I., FlitsCh, R., IBM J. Res. Dev. 22 (1978) 294-303.

[17] Harrington, W. L., Appl. Phys. Lett. 27 (1975) 664 669.

[18] Lalov, I. J., Modern problems of surface physics, Bulg. Acad. Sci., Sofia (1981).

[19] Hollinger, G., Appl. Surf. Sci. 8 (1981) 318-336.

[20] Romanov, O. V., Sokolov, M. A., SultanmagoMEDOV, S. N., 3e Colloque sur la physico-chimie des surfaces de semiconducteurs, Acad. Sci. d'URSS, Novosibirsk (1978).

[21] Romanov, O. V., Sokolov, M. A., Konorov, P. P., ANDREEV, A. D., Micro-electron. (en russe) 6 (1977) 340-347.

[22] Romanov, O. V., Sultanmagomedov, S. N., Vestnik Leningradskogo Universiteta (en russe), phys.chem. 2 46-52 (1980).

[23] Romanov, O. V., Ribeiro de Silva, M. K., Mikroelektron. (en russe) 4 (1975) 269-274.

[24] Romanov, O. V., Iafassov, A. M., Rudenko, M. I., Sov. Phys. Semiconductors 9 (1975) 325-327. 